\title{
COVID-19: Guidance Outlines on Infection Prevention and Control for Health Care Workers
}

\section{Suresh Kumar Sharma ${ }^{1}$, Shiv Kumar Mudgal ${ }^{2}$, Prasan Kumar Panda ${ }^{3}$, Pratima Gupta ${ }^{4}$, Pradeep Aggarwal ${ }^{5}$}

${ }^{1}$ Professor \& Principal, College of Nursing, All India Institute of Medical Sciences, Rishikesh, Uttarakhand, India; ${ }^{2}$ Nursing Tutor; College of Nursing, All India Institute of Medical Sciences, Rishikesh, Uttarakhand, India; ${ }^{3}$ Assistant Professor; Department of Medicine; All India Institute of Medical Sciences, Rishikesh, Uttarakhand, India; ${ }^{4}$ Professor \& Head, Department of Microbiology, All India Institute of Medical Sciences, Rishikesh, Uttarakhand, India; ${ }^{5}$ Associate Professor, Department of Community \& Family Medicine, All India Institute of Medical Sciences, Rishikesh, Uttarakhand, India

\begin{tabular}{|c|c|c|c|c|c|c|c|}
\hline Abstract & Introduction & Methodology & Results & Conclusion & References & Citation & ables / Figures \\
\hline \multicolumn{8}{|c|}{ ling Author } \\
\hline \multicolumn{7}{|c|}{$\begin{array}{l}\text { Prof Suresh K Sharma, PhD, RN, Professor \& Principal, College of Nursing, All India Institute of Medical } \\
\text { Sciences, Rishikesh, Uttarakhand, India } \\
\text { E Mail ID: skaiims17@gmail.com }\end{array}$} & 口is \\
\hline
\end{tabular}

\section{Citation}

Sharma SK, Mudgal SK, Panda PK, Gupta P, Aggarwal P. COVID-19: Guidance Outlines on Infection Prevention and Control for Health Care Workers. Indian J Comm Health. 2020;32(1):08-14.

Source of Funding: Nil Conflict of Interest: None declared

\section{Article Cycle}

Received: 18/02/2020; Revision: 22/02/2020; Accepted: 01/03/2020; Published:04/03/2020

This work is licensed under a Creative Commons Attribution 4.0 International License.

Coronavirus are a common family of viruses and there are seven different types of coronaviruses including this new member of coronavirus i.e. 2019 novel coronavirus, which can make the people infected and sick. Some strains of this family of virus cause mild to moderate disease symptoms like common cold while other strains cause very severe disease like Severe Acute Respiratory Syndrome (SARS) and Middle East respiratory syndrome (MERS).(1,2)

In late December, in Wuhan Hubei Province, China a number of people suffered with severe respiratory illness including a cluster of pneumonia cases. On 31 December 2019, China notified the World Health Organization (WHO) about cluster of patient with symptoms of respiratory illness of unknown cause, which were connected to an open seafood and animal market of Wuhan city, China.(3)

On 12 January 2020 after analysis of virus genetic sequence in sample collected from patient, it was declared that a novel coronavirus had been the cause of this epidemic. This new virus is named as '2019 Novel Coronavirus' and the disease related to this is termed as '2019-nCoV acute respiratory disease' (where ' $n$ ' stand for novel and 'CoV' for coronavirus). Later on, WHO has suggested it should be named as 'Sever Acute Respiratory Syndrome' (SARS-CoV-2) and the diseases as 'COVID2019' (Corona Virus Infection Disease 2019). China and WHO shared information that new coronavirus is a betacoronavirus which has akin genetics to SARS-like coronavirus. After full-genome sequencing and phylogenic analysis determined that SARS-CoV-2 is a different clade from the beta coronaviruses associated with human SARS and MERS. $(4,5)$

As on $4^{\text {th }}$ March 2020, WHO reported globally 93,090 laboratory confirmed (76 countries, 2223 new in last 24 hours) cases while in China 80,422 (120 new) cases were reported; where 2984 mortalities ( 38 new) in China and 214 deaths (48 new) mortalities in rest of the world have been reported. In India six confirmed cases were reported (one new cases) with no mortality (5).

\section{Transmission}

As coronaviruses are a big family of viruses which are prevalent in many distinct categories of animals, like camels, cattle, cats, and bats. Hardly, animal coronaviruses can pass infection to human and then transmit from human-to-human like MERS, SARS, and now with SARS-CoV-2.(4)

The source of this coronavirus outbreak has not been determined yet. Illegal connection of bat is suspected to be the source for entrance of Covid-19 in to human. Initial investigations identified positive sample for COVID-19 cases who visited to Seafood and Animal Market in Wuhan City; therefore, the source of the infection has been associated to this. However, some patients who were diagnosed by laboratory reported no exposure to this market. A zoonotic origin to this epidemic has to identify yet. Although evidence is continuously coming up, fact till date suggests human-to-human spreading. $(3,6)$ 
Much is unknown about the spread of SARS-CoV-2. Recent knowledge is mainly depending on what is already known about similar coronaviruses. Based on what is currently known about SARS-CoV-2 and what is known about other coronaviruses with epidemic potential like MERS and SARS, spread is thought to occur mostly from person-toperson via respiratory droplets among close contacts and direct or indirect exposure to infected sources. It is unclear till now that a person can get infection of SARSCoV-2 if he/she touch a surface or item that has infected and then touch his/her own mouth, nose, or eyes. $(3,4,5)$ Information from recent study suggest that infection of SARS-CoV-2 could be transmitted from the people before they present the symptoms. (7)

\section{Clinical Presentation}

As per CDC believes; based on what has been experienced and reported about the incubation period of MERS; SARSCoV-2 may show its symptoms from 2 days to 2 weeks after exposure. Symptoms include fever, cough and shortness of breath. Chest X-ray may present bilateral infiltrates.(4) Disease may range from mild to severe level of symptoms and death. (5)

Recent case studies reported the most common symptoms at the onset of disease were fever, cough, myalgia or fatigue and shortness of breath among $83-98 \%$, $46-82 \%, 11-44 \%$ and $31 \%$ patients respectively. Some patients also reported sore throat in early stage of illness while symptoms of sputum production, headache, hemoptysis, and diarrhea were reported less commonly $(8,9,10)$. Case definitions of surveillance has been illustrated in Box-1.

Box 1: Case definitions for surveillance $(4,5)$

1. Suspected a. Person who had acute respiratory Case illness, met at least one of the following symptoms - sudden onset of sore throat, cough and dyspnea; AND with no other etiology that fully explains the clinical presentation and a history of travel to or residence in a country/ area/ territory reporting local transmission of COVID-19 disease during the 14 days prior to symptom onset.

OR

b. A person with any acute respiratory illness AND having been in contact with a confirmed or probable COVID-19 case in the last 14 days prior to onset of symptoms. OR

C. A person with sever acute respiratory infection (fever and at least one sign/ symptom of respiratory disease eg. cough, shortness of breath) AND requiring hospitalization AND with no other etiology that fully explains the clinical presentation.

2. Probable A suspected person for whom the case results of investigations for COVID19 by the laboratory is not conclusive or for whom investigation was positive on a pan-coronavirus assay.

3. Confirmed A person for whom the reports of case laboratory testing for COVID-19 confirm infection, irrespective of clinical presentation of the person.

\section{Definition of Close Contact}

Close contact can take place when providing care for, staying with, visiting, or sharing a common waiting area or room with a COVID-19 person. A close contact is defined as a person who is staying in the same household or being within approximately six feet of the case. A contact in health care facility defined as a person who provided direct care to the person infected with SARS-CoV-2 or dealing with his/her body fluids or sample or has been in the same area when an aerosol generating procedure has been carried out. A contact in an aircraft defined as a person seated within two seats (either right or left side) of the COVID-19 patient, companion who provided care to the COVID-19 patient, and crew members providing their service to the patient. $(6,7)$

\section{Guidance Outlines on SARS-CoV-2 Infection Prevention} and Control Precautions $(6,7,12-16)$

This paper tries to explain guidance outlines on infection prevention and control (IPC) precaution to be used by heath care professionals, when they are caring patients with suspected or confirmed SARS-CoV-2 infection. These guidance outlines should be practiced in agreement with local or nation guidelines.

This guideline is still under review and based on the limited scientific fact about SARS-CoV-2/ COVID-19 as it is a recently detected strain of coronavirus family. Therefore, this guidance is based on previous knowledge about coronaviruses with serious epidemic potential like MERS and SARS and on recent information of the situation in China and other countries where cases were identified. This judicious attempt will be refined and revised as further scientific information becomes accessible about SARS-CoV-2.

As it is well-known fact that SARS and MERS can pass on person to person; although this is still unclear about SARSCoV-2, it is fair to presume that human-to-human transfer is feasible.

As till now there is no effective drug or a vaccine against this virus, management of this epidemic depends on effective prevention and control of disease transmission. These preventive actions include use transmission-based precautions (e.g. precautions for contact, droplet and 
airborne) with the effective implementation and ensuring IPC measures in health care facilities. These measures include:

\section{Preparedness}

In preparation, $\mathrm{HCW}$ s or health care setting involved in the diagnostic testing, treatment and care for suspected, probable or confirm COVID-19 patients should:

- Review and understand the local and national protocols and confirm that standard operational procedures (SOPs) are explained clearly, and HCWs are informed with them; e.g., how PPE should be used and where PPE is stored.

- Review SOPs for promptly and effectively decontaminating areas of the facility where suspected or confirm cases have been placed.

- Ensure that HCWs are familiar to that area where a case will be isolated and the requirement of an Airborne Infection Isolation Room (AIIR). It is a room at negative pressure with a minimum of 12 air change/hour and accommodate only single patient. Air before recirculation should be filtered through a high-efficiency particulate air (HEPA) filter or directly exhausted to outside.

- Ensure that HCWs involve in assessment or care for COVID-19 patients should be well aware with a Filtering Face-piece respirator (FFR), and undertake a fit test to ensure proper fit every time before using this device. If HCWs cannot use a FFR respirator because of improper fit, then powered hood respirator or other alternative with equivalent protection should be used.

- Ensure that HCWs who are caring for COVID-19 case should be well trained in the proper donning and doffing with adequately disposal of PPE. As it is reported from the studies that HCWs are more likely to use PPE improperly and incorrectly. Therefore, they put themselves at high risk of getting infected. So facilities will need not only train their HCWs but also provide continuous re-enforcement.

- Ensure HCWs are aware to whom they should contact for discussion and information about a suspected, and probable case within their health care facility.

- Ensure that sufficient and appropriate supplies of Filtering Face Piece-3 (FFP3) respirators, gloves, fullsleeved, disposable gown, eye protection (e.g. disposable goggles or face visor), biomedical waste bins, hand wash material, natural detergent and chlorine based solution for disinfection etc. and all HCWs should be well trained regarding the proper use of these supplies or equipment needed while working with patients. For this, administration's commitment, accountability, and a clear policy are required.

\section{Appropriate and prompt triage system}

Triage system includes prompt and correct assessment of all patients at the time of admission which facilitate early detection, and control of transmission (isolating patients with suspected case of COVID-19). For the early detection of cases of suspected COVID-19, healthcare setting should:

- Encourage HCWs to screen all patients visiting the hospital with clinical suspicion.

- Set up an advanced and well equipped station at the entrance of health care setting, supported by well and specially trained HCWs.

- Implement the use of screening questionnaires based on the updated case definition of COVID-19.

- Paste visual alerts with specific instructions in appropriate language at specific places like entrance, waiting areas, lifts, canteen etc. about preventive measures of SARS-CoV-2.

- Encourage all persons including HCWs for hand hygiene, respiratory hygiene, cough etiquettes and appropriate use of PPE.

- Ensures fewer crowds.

III. Standard precautions for all patients

Standard precautions should be used believing that each individual is likely to have infection with an organism that could be spread in the healthcare facility. Instruct, encourage and supervise all HCWs, patients and their attendants to practice standard precautions which include:

- Hand hygiene:

- Follow the WHO's 'My 5 Moments for Hand Hygiene approach' (before touching a patient, before performing any procedure, after exposure to body secretion, after touching a patient, and after contacting the surrounding or items of a patient).

- Perform hand hygiene preferably with an alcoholbased hand rub (ABHR) if hands are not visibly soiled or with soap and water if visibly soiled.

- Respiratory hygiene and cough etiquette: It will minimize the risk of cross-infection of respiratory disease and include the following measures:

- All person should be encouraged to practice to use disposable tissue to cover their nose and mouth when sneezing and coughing

- all used tissues should be discarded immediately into a proper biomedical waste bag

- Personal protective equipment (PPE): instruct, reenforce and supervise all persons and HCWs for correctly and consistently use of PPE (glove, gown, respirator and eye protection).

- Use N-95 respirators by all individual entering the patient's area. HCWs must be fit tested before applying this respirator. Virus particle size is believed to be large enough, so if $\mathrm{N}-95$ mask is not available, simple surgical face mask also may be used.

- Use of full-sleeved, fluid-resistant and single use gown. 
- Use disposable gloves when contact with the patient or surroundings.

- Use eye protection when contact with patient.

- Clinical waste must be discarded in leak-proof biomedical waste bin and disposed properly.

- Used linen must be categorized as contaminated.

- $\quad$ Effective and proper cleaning and disinfection of the environment is also very much needed to prevent the transmission of SARS-CoV-2.

\section{Specific measures for care of COVID-19 suspected and} confirmed cases

1. Patient placement (Isolation)

- A suspected or confirm case should be placed in an AlIR if available. A single with attached toilet and bathroom should be used when an AlIR is not available. Room doors should be kept closed

- In a critical care unit (CCU), the case should be managed in a single cubical with negative-pressure if possible, or, in a neutral-pressure room with the door closed when negative pressure is not available.

- If room with attached toilet facility is not available, then an exclusive assigned commode should be used. It should be cleaned and decontaminated as per recommended protocol or schedule. Appropriate arrangements should be done for the safe removal of the bedpan.

- Avoid keeping any unnecessary or extra items or equipment in the patient's room.

2. Lobbies (anterooms) and donning and doffing PPE

- Lobbies also have the likelihood to get contamination and should be cleaned and disinfected regularly as per environmental disinfection policies.

- It is strongly suggested that HCWs move from 'dirty' to 'clean' zones within the lobbies as they remove their PPE and perform hand hygiene after they exit from the room. Any extra items should not be store in lobby area.

- A peer system to notice for unintentional contamination is advised, especially when high risk procedures performed and during the removal of PPE.

3. Information about infection risks

- Information about the requirement of isolation and infection prevention control measures which must be undertaken before entering the patient' room must be placed on the door of isolation room. It is strongly advised that confidentiality of the patient must be maintained.

4. Staff considerations

- $\quad$ All HCWs should be aware about emergency contact details involved in patient care so that if they get symptoms of COVID-19 can contact to health care facility immediately.

5. Visitors

- All visitors should be restricted to essential visitors only; such as parents of pediatric patients or an
[Corona Virus...] | Sharma SK et al affected patient's main career. This should be subject to a local risk assessment having been performed.

- It is suggested to screen all visitors for symptoms before entering the healthcare setting.

- $\quad$ Alternative ways for patient and visitor interactions (e.g. video-call) should be utilized.

- PPE must be made available to visitors, including instruction, demonstration and supervision of correct usage and donning and doffing.

6. Personal Protective Equipment(16)

- All persons entering the patient room should wear PPE. Donning and doffing of PPE must be done in the sequence as given below (Box-2):

Box-2: Sequence of donning and doffing of the Personal Protective Equipment (PPE)

DONNING DOFFING

Full-sleeves gown remove gloves and dispose in proper biomedical waste bin and wash hand thoroughly

FFP 3 respirator Taking out gown by using a and done fit- peeling motion, fold gown in on checked itself and place in biomedical waste bin

Eye protection remove goggles or visor only by (single use googles the headband or sides and discard or full-face visors) in biomedical waste bin

Disposable gloves Putting off respirator from behind with tight fitting and discard in proper biomedical cuff waste bin Wash hand thoroughly.

- Before donning, healthcare workers should put on scrubs, ensure hair is tied back securely and off the neck and collar, remove jewelry or pens, ensure they are hydrated, and perform hand hygiene. During donning each item must be adjusted as required to ensure it fits correctly and interfaces well with other PPE items.

- It is advised that gloves, gown and eye protection should be removed prior to leaving patient room and disposed them biomedical waste bin. After leaving the area, the respirator can be put off and disposed in proper biomedical waste bin after leaving the room.

- It is very much crucial to understand that order for donning (putting on) of PPE is less critical then the order for doffing (putting off) to minimize the risk of cross contamination.

7. Hand hygiene

- This is very much effective step and perform it before and after contact to each patient, removal of PPE, contact with patient surrounding or items and disinfection of the environment.

- Perform hand wash with soap and water or use an alcohol hand rub when hands are visibly clean. 
- It is strongly advised to all HCWs that they must not wear rings (expect a plain smooth band), wrist watches and jewelry.

8. Aerosol generating procedures

- Any procedure that generates aerosols, such as open suctioning, induced cough, bronchoscopy, intubation, extubation and positive-pressure ventilation via a face mask, have a high risk of spread. When these procedures are medically needed, they should be carried out in an AlIR, if available or in a single room with the door closed.

- Only the essential and well trained HCWs should be present in procedure room, and all must wear PPE as per recommendation. It is advised to minimized entry and exit from the room during the procedure.

- When an aerosol producing procedure is carried out in the patient's room, then same room should be disinfected 20 minutes after the completion of procedure (as after 20 minutes there would be less than $1 \%$ of aerosols then the starting load).

- When an another room is utilized for an aerosol producing procedure room should be left for 20 minutes, then decontaminate prior to use it again.

9. Use of equipment

- As far as possible reusable supplies, items (e.g. crockery\& cutlery, shaving kit etc.) and equipment should be avoided to minimize the risk of cross contamination and load of items which require be cleaning and disinfecting.

- As per hospital waste management policy, all single use items or equipment must be disposed.

- Re-useable equipment or supplies; if used, it should be cleaned and disinfected as per the instructions of manufacturer prior to leave the room.

- Use exclusively assigned supplies to the patient in the isolation room.

- Use high efficiency filter to protect ventilators.

- Use closed suction system for all cases.

10. Environmental cleaning and decontamination

- It is suggested that that a well-trained domestic staff should perform cleaning and decontamination with use of the proper PPE.

- After cleaning with natural detergent, it should be decontaminated by a chlorine based disinfectant solution with a minimum strength of $1000 \mathrm{ppm}$ chlorine availability or as per policy of heath care facility.

- The patient room should be cleaned and decontaminated at least once a day.

- Commonly used hand touch surfaces and lobbies should be cleaned at least twice in a day.

- It is strongly advised that cleaning and decontamination of the isolation areas is performed separately to the cleaning of other areas.

- If possible environmental decontamination must be done by disposable equipment. Reusable equipment must be dedicated to particular area and disinfected after use with a chlorine-based disinfectant or solution recommended by the facility.

11. Linen

- Leak proof linen bag should be kept inside patient room as per policy for infectious linen.

- Un-bagged linens should not be transferred through the ward or other areas or clinical wards.

12. Waste management

- $\quad$ Large amount of waste may be produced by frequent utilization of PPE; get and follow the guidelines from the hospital waste management team to manage this issue.

- All wastes should be disposed as per accordance for infectious clinical waste.

- All waste from a suspected or confirmed case strictly disposed as per Category B waste management.

- An ambulate patient can use the attached facilities. If patient used bedpans, use super absorbent polymer gel granules for solidification of excreta and then disposed it as hospital waste. It is suggested to avoid communal facilities.

13. Specimens

- All samples and request forms should be marked with a biohazard label.

- The samples should be double-packed in the same room where sample has been collected by a HCW wearing PPE.

- All sample should be handed over personally to the laboratory by a person who understands the nature of the samples. It is strongly suggested to avoid pneumatic tube systems to transport sample.

- Transport of specimens between laboratories should be as per Category B transportation recommendations.

14. Use of mobile equipment

- Use of mobile devices like portable ECG, X-ray machines etc. should be restricted to needed requirements.

- The technician who are not involve in the patient care routinely, must be instructed and supervised in infection prevention precautions, like proper use of PPE.

- The technician should use PPE when enter the patient room.

- $\quad$ Any mobile device taken in to the patient area must be cleaned and decontaminated before leaving the lobby as per policies.

- In addition to device any additional items like a digital detector or a cassette should be decontaminated, regardless of their direct contact with the patient.

15. Critical care

- A high efficiency filter must be used to protect all respiratory devices or equipment. It is suggested to dispose these filters after use. 
- As far as possible single use respiratory equipment should be used. If not possible, re-usable equipment must be cleaned and disinfected as per instructions of manufacturer prior to re-use.

- A closed system for suctioning should be used

- Circuits of ventilator should not be broken unless needed.

- When carrying out bagging, ventilators must be kept on standby mode.

- $\quad$ All HCWs must wear appropriate PPE.

- It is suggested to avoid water humidifier, use heat and moisture exchanger.

16. Transfers of patient to other departments

If possible, all diagnostic testing and procedures should be performed in the single negative pressure room with the presence of minimum and essential HCWs. patients should be transferred to other departments only presence of specific clinical needs and after detail discussion with infection control team. The transfer of patient to other department includes following procedures:

- The bed or trolley that utilized to transfer the patient from the room of one department to other department should be decontaminated immediately prior to leaving the patient room by a HCW with protective cloths and PPE.

- The department where the patient is going to be transferred must be informed in advance.

- Any unnecessary equipment or item must be removed from the procedure or treatment room.

- The patient must be shifted directly to and from the procedure or treatment room and must not be kept in a common area.

- The procedure or treatment room, bed, trolley or chair and other used equipment should be cleaned and disinfected immediately after use.

- Ensure that anyone involved in performing procedure, shifting the patient or staying within 6 feet must wear the PPE.

17. Transfers the patient to other facilities

- Transfer the patient to other health care facility should be restricted unless it is deemed needed for medical care.

- The facility and the ambulance staff where the patient is going to be transferred must be informed in advance about the transfer of patient, so that specific infection prevention measures can be initiated.

18. Caring dead bodies

- A body bag should be used for transferring the body should be transferred in a body bag and HCWs who involved in handling the body must use PPE.

- As per environmental disinfection protocols decontaminate the outer surface of the body bag and the trolley carrying the body immediately prior to body bag exit from the lobby. This process requires minimum two person wearing all PPE and protective cloths.
- HCWs must remove their protective clothing before leaving the lobby.

- HCWs should use all PPE when it is needed to open the body bag in the hospital mortuary.

- It is acceptable to wash or prepare with wearing full PPE. Mortuary and funeral persons must be informed of the biohazard risk.

- When post mortem is needed; it is advised to use safe working techniques (for example manual rather than power tools) with wearing full PPE. If needed use high security posts mortem suites.

- Empty body bags must be disposed of as per regulation of Category-B waste management.

\section{Using environmental and engineering controls}

- These controls address the basic infrastructure of the health care facility.

- These controls aim to ensure there is adequate ventilation in all areas in the healthcare facility, as well as adequate environmental cleaning. Additionally, isolation of all patients is needed. Both isolation and adequate ventilation can minimize the spread of infection in the healthcare setting. Air circulation can be managed in such a way that infections should not be spread inside the hospital and properly drained to environment with least possible transmission. Ensure that cleaning and disinfection should be done regularly and strictly as per guidelines.

- Ensure laundry, food services and biomedical waste in accordance with safe routine procedures.

\section{Reporting within healthcare setting and to local health authorities}

- Implement policies that promptly inform local or state health authorities about suspected or confirm case of COVID-19.

- It is suggested to designate team or person from the facility who are responsible for collecting and dissemination of information to public health authorities and HCWs within facility.

\section{Conclusion}

Source of SARS-CoV-2 outbreak is believed to be bat with possibilities of human-to-human transmission by direct contact and droplet infection. It has no definitive treatment and vaccination, except a hope from new antivirals. Therefore, prevention and control of infection is the key to combat this health problem; which includes revised latest updated SOP of SARS-CoV-2 infection prevention and control, training of HCWs, appropriate use of PPE, regular hand hygiene, appropriate waste management, respiratory and cough etiquettes, regular environmental cleaning and decontamination, hospital visitor's education and control etc. 


\section{References}

1. Li $Q$, Guan $X$, Wu $P$, Wang $X$, Zhou L, Tong $Y$, et al. Early transmission dynamics in Wuhan, China, of novel coronavirus-infected pneumonia. N Engl J Med. 2020 Jan 29. doi: 10.1056/NEJMoa2001316.

2. Department of Health and Social Care. GOV.UK. https://www.gov.uk/government/organisations/departme nt-of-health-and-social-care; 2020. [last accessed on 18/2/20]

3. Zhu N, Zhang D, Wang W, Li X, Yang B, Song J, et al. A novel coronavirus from patients with pneumonia in China, 2019. N Engl J Med 2020 Jan 24. doi: 10.1056/NEJMoa2001017.

4. CDC. 2019 Novel Coronavirus (2019-nCoV). Centers for Disease Control and Prevention. https://www.cdc.gov/coronavirus/2019-ncov/hcp/clinicalguidance-management-patients.html; 2020. [last accessed on $18 / 2 / 20$ ]

5. WHO. Novel Coronavirus (2019-nCoV) situation reports. https://www.who.int/emergencies/diseases/novelcoronavirus-2019/situation-reports; 2020. [last accessed on $04 / 3 / 20$ ]

6. COVID-19: investigation and initial clinical management of possible cases. GOV.UK.

https://www.gov.uk/government/publications/wuhannovel-coronavirus-initial-investigation-of-possiblecases/investigation-and-initial-clinical-management-ofpossible-cases-of-wuhan-novel-coronavirus-wn-covinfection; 2020. [last accessed on 18/2/20]

7. 2019 Novel Coronavirus (2019-nCoV) [Internet]. Centers for Disease Control and Prevention. https://www.cdc.gov/coronavirus/2019-ncov/hcp/hcppersonnel-checklist.html; 2020. [last accessed on 18/2/20]

8. Huang C, Wang Y, Li X, Ren L, Zhao J, Hu Y, et al. Clinical features of patients infected with 2019 novel coronavirus in
Wuhan, China. Lancet 2020 Jan 24. pii: S01406736(20)30183-5. doi: 10.1016/S0140-6736(20)30183-5.

9. Wang D, Hu B, Hu C, Zhu F, Liu X, Zhang J et al. Clinical Characteristics of 138 Hospitalized Patients With 2019 Novel Coronavirus-Infected Pneumonia in Wuhan, China. JAMA. 2020 Feb 7.doi: 10.1001/jama.2020.1585.

10. Chen N, Zhou M, Dong X, Qu J, Gong F, Hen Y, et al. Epidemiological and clinical characteristics of 99 cases of 2019 novel coronavirus pneumonia in Wuhan, China: a descriptive study. Lancet. 2020 Jan 30. pii: S01406736(20)30211-7. doi: 10.1016/S0140-6736(20)30211-7.

11. European Centre for Disease Prevention and Control. https://www.ecdc.europa.eu/en/home; 2020. [last accessed on 18/2/20]

12. Patient management. Who.int. https://www.who.int/emergencies/diseases/novelcoronavirus-2019/technical-guidance/patientmanagement; 2020. [last accessed on 18/2/20]

13. Novel Coronavirus-2019: What Nurses Need to Know National Nurses United. https://www.nationalnursesunited.org/2019-nCoV; 2020. [last accessed on 18/2/20]

14. Coronavirus: RCN publishes resource for nurses | Nursing in Practice. https://www.nursinginpractice.com/vaccinationsinfections/coronavirus-rcn-nurses-resource-uk; 2020. [last accessed on 18/2/20]

15. What to do if you suspect coronavirus in your practice | Nursing in Practice. https://www.nursinginpractice.com/vaccinationsinfections/what-do-if-you-suspect-coronavirus-yourpractice; 2020. [last accessed on 18/2/20]

16. Wax R S, Christian MD. Practical recommendations for critical care and anesthesiology teams caring for novel coronavirus (2019-nCoV) patients. Can J Anaesth2020Feb 12. doi: 10.1007/s12630-020-01591-x. 